\title{
円環状き裂を有する無限体の非定常熱空力*
}

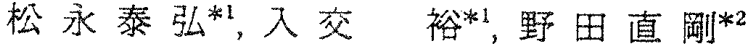

\section{Transient Thermal Stress in an Infinite Elastic Solid Containing an Annular Crack}

\author{
Yasuhiro MATSUNAGA, Hiroshi NYUKO, and Naotake NODA
}

\begin{abstract}
The present paper seeks to solve the transient thermal stress in an infinite, isotropic solid containing an annular crack. It is assumed that the transient thermal stress is set up by the applica. tion of the heat exchange by convection on the crack surface. By use of the finite difference method for only the time variable, the analytical solution for spatial variables can be obtained. The numerical results are shown for the temperature and stress intensity factor.
\end{abstract}

Key Words: Elasticity, Thermoelasticity, Infinite Solid, Annular Crack, Unsteady State, Axial Symmetry

\section{1. 緒言}

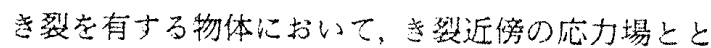

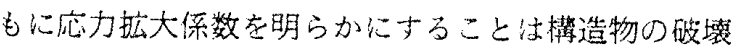
を推定するうえで重琶である。气のため，き裂を有文 る物体の熱応力法数多く報告きれているが，大多数仙 定常熱灾力に関してである。非定常熱応力については,

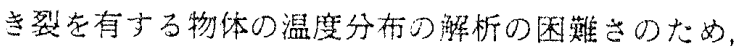
あまり報告されていない(()ー(4)。

そこで，理論解析の困難なき裂を有なる非定常熱伝 導問題に対し，著者らは時間变数のみ心階差法を用い ることにより架間変数に対して解析解を得る方法を提 案し，直線き裂を有する平板，円形き裂を有する無限 体，外部き裂有する無限体の非定常熱応力 ${ }^{(5) \sim(7)}$ の解 析を行っている。

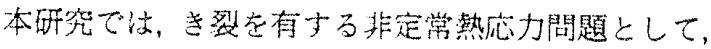
非定常熱伝薯の解析心は著者うの提婪した力法を用い

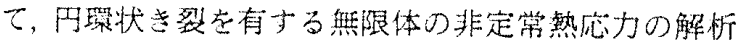
を行った。た，数㒹計算を宾行し，温度分布㧍上じ応

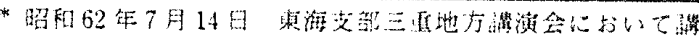
演。激稿受付昭和 62 年1月14 1 .

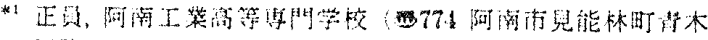
265).

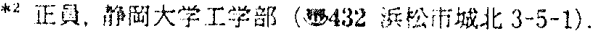

力法大係数について図示した。

\section{2. 解析}

$2 \cdot 1$ 温度分布 図1に示すように円疅状き列を

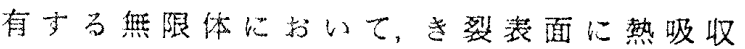
一 $q_{0} f(r) g(t)$ (ただし，

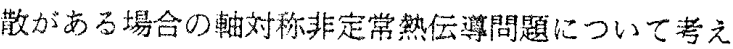
る。

\section{熱伝邀の基磱方方程式流}

$$
\nabla^{2} T=T_{, 2} / x
$$

ただし、 $\nabla^{2}=\partial^{2} / \partial r^{2}+r^{-1} \partial / \partial r+\partial^{2} / \partial z^{2}, x$ は熱詓散率， $T$ は温度, $T_{t} \equiv \partial T / \partial t$ でる。

初期条件，境瑟条件は

$$
t=0 \text { て } T=0 \text {. }
$$

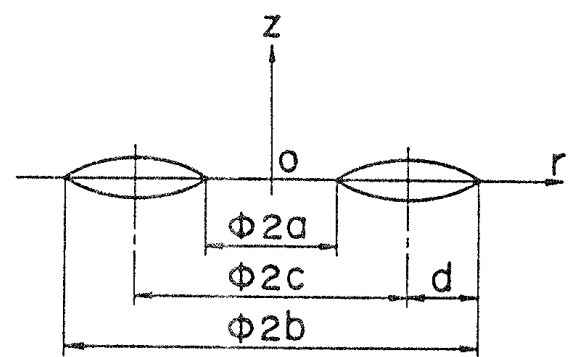

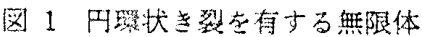




$$
\begin{aligned}
& z=0 \tau \quad T_{. z}=\left\{h T-\left[-q_{0} f(r) g(t) / \lambda\right]\right\} \\
& \times H(r-a) H(b-r)
\end{aligned}
$$

て与员うれるとする。ただし，んは相効熱伝達率，入は 熱伝導率, 一 $q_{0}$ は一定な急の発熱量, $H(）$ 仕へビサ イドのステップ関数である。

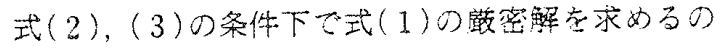
は困難であるので，時間に対してのみ階荎法を用いて 解析する。そこで，任意時間 $t_{j}$ と時間きさか $\Delta t$ 死

$$
t_{j}=\sum_{k=1}^{j} \Delta t_{k}, \Delta t_{j}=t_{j}-t_{j-1}
$$

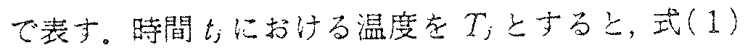
の階差表示放

$$
\nabla^{2} T_{j}-\frac{1}{x \Delta t_{j}} T_{j}=-\frac{1}{x \Delta t_{j}} T_{j}
$$

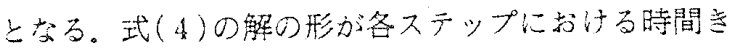
ざみ $\Delta t_{j}$ の值のとり方に依存するので， $\Delta t_{j}$ がすべて 晎なる場合心ついて考える。

式（A）の一般解法補助解 $T_{j c}$ と特解 $T_{j p}$ の和

$$
T_{j}=T_{j c}+T_{j p}
$$

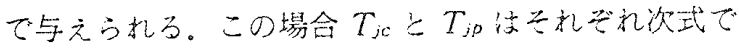
与えられる。

$$
\begin{aligned}
& T_{j c}=\int_{0}^{\infty} A_{j} p J_{0}(p r) e^{-s_{j} z} d p \\
& T_{j p}=\sum_{k=1}^{j-1} C_{j k} T_{h c}
\end{aligned}
$$

たたし，Jい）は箩1種レ次のベッセル関数で,

$$
\begin{aligned}
& C_{j k}=\prod_{m=k+1}^{j} \frac{\Delta t_{k}}{\Delta t_{k}-\Delta t_{m}} \\
& s_{k}=\sqrt{p^{2}+1 / x \Delta t_{k}}
\end{aligned}
$$

式(5)在式（3）に代入すると，三雷連立䖽行方程式

$$
\left\{\begin{array}{c}
\sum_{k=1}^{j} C_{j k} \int_{0}^{\infty} A_{k} s_{k} p J_{0}(p r) d p=0 \\
(0 \leq r<a, b<r<\infty) \\
\sum_{k=1}^{j} C_{j k} \int_{0}^{\infty} A_{k}\left(s_{k}+h\right) p J_{0}(p r) d p=-\frac{q_{0}}{\lambda} f(r) g\left(t_{j}\right) \\
(a<r<b)
\end{array}\right.
$$

在得る。式 $(8)$ 変形一ると

$$
\left\{\begin{aligned}
& \int_{0}^{\infty} A_{j} s_{j} p J_{0}(p r) d p=0 \\
&(0 \leq r<a, b<r<\infty) \\
& \int_{0}^{\infty} A_{j}\left(s_{j}+h\right) p J_{0}(p r) d p=-\frac{q_{0}}{\lambda} f(r) E_{j} \\
&(a<r<b)
\end{aligned}\right.
$$

ただし，

$$
E_{j}=g\left(t_{j}\right)-\left(1-\hat{o}_{j 1}\right) \sum_{k=1}^{\prime-1} C_{j k} E_{k}
$$

第 1 式を恒等的に满足主るように，东知係数 $A_{j}$ 学

$$
A_{j}=-\frac{q_{0}}{i} \frac{1}{s_{j} p} \sum_{n=1}^{\infty} A_{j n} Z_{n}(p)
$$

とちく( ${ }^{(8)}$ 。ただし

$$
\left.\begin{array}{l}
Z_{n}(p)=p\left\{G_{n-1}(p)-G_{n+1}(p)\right\} \\
G_{n}(p)=J_{n}(p c) J_{n}(p d)
\end{array}\right\}
$$

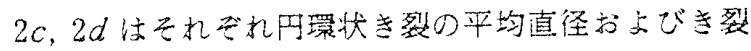
の幅であり

$$
c=(a+b) / 2, d=(b-a) / 2
$$

て表される。式(11)を式(9)0第2式心代入方ると，

$$
\begin{gathered}
\sum_{n=1}^{\infty} A_{j n} \int_{0}^{\infty}\left(1+\frac{h}{s_{j}}\right) Z_{n}(p) J_{0}(p r) d p=f(r) E_{j} \\
(a<r<b)
\end{gathered}
$$

ここで, 変数 $r を$

$$
r=\sqrt{c^{2}+d^{2}-2 c d \cos \phi}
$$

により新しい変数 $\phi$ に変換すれ证変域 $a \leq r \leq b$ 洨 0 $\leq \phi \leq \pi(r=a て ゙ \phi=0, r=b て ゙ \phi=\pi)$ にる。ノイ

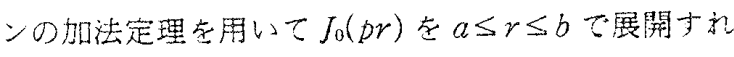
i゙

$$
J_{0}(p r)=G_{0}(p)+2 \sum_{i=1}^{\infty} G_{i}(p) \cos i \phi
$$

また，f(r)をフーリエ級数に䦙開すると，

$$
f(r)=f_{0}+2 \sum_{i=1}^{\infty} f_{i} \cos i \phi
$$

となる。たたし，

$$
\begin{gathered}
f_{i}=\frac{1}{\pi} \int_{0}^{\pi} f\left(\sqrt{c^{2}+d^{2}-2 c d \cos \phi}\right) \cos i \phi d \phi \\
(i=0,1,2, \cdots)
\end{gathered}
$$

式(14)，(15) 式(13)に代入すると

$$
\begin{aligned}
& \sum_{n=1}^{\infty} A_{j n} \int_{0}^{\infty}\left(1+\frac{h}{s_{j}}\right) Z_{n}(p)\left\{G_{0}(p)\right. \\
& \left.+2 \sum_{i=1}^{\infty} G_{i}(p) \cos i \phi\right\} d p \\
& =\left\{f_{0}+2 \sum_{i=1}^{\infty} f_{i} \cos i \phi\right\} E_{j}(0<\phi<\pi)
\end{aligned}
$$

を得る。式(16)は任意の申について成立しなければな らないから，闻辺の係数をしく等しくと末知係数 $A_{j n}$ 块定支热限連立一次方程式

$$
\begin{aligned}
& \sum_{n=1}^{\infty} A_{j n} \int_{0}^{\infty}\left(1+\frac{h}{s_{j}}\right) \frac{1}{p} Z_{n}(p) Z_{i}(p) d p \\
& =\left(f_{i-1}-f_{i+1}\right) E_{j} \\
& \quad(i=1,2,3, \cdots) \cdots
\end{aligned}
$$

在得吕。したがって，温度分布 $T_{j}$ は

$$
T_{j}=-\frac{q_{0}}{i} \sum_{k=1}^{j} C_{j k} \sum_{n=1}^{\infty} A_{k n} \int_{0}^{\infty} \frac{1}{s_{k}} Z_{n}(p) J_{0}(p r) e^{-s_{n} z} d p
$$

で方放れる。

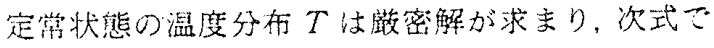


与えられる。

$$
T=-\frac{q_{0}}{\lambda} \sum_{n=1}^{\infty} A_{\infty n} \int_{0}^{\infty} \frac{1}{p} Z_{n}(p) J_{0}(p r) e^{-p z} d p
$$

ただし， $A_{\infty n}$ 洨式(17)で $s_{j}, E_{j}$ をれてれ に置き換之心無限連立一次方程式の解である。

次に $h=00$ 持別な場合，士なわら，裂表面から 時間と位置の関数の熱流束が流出する場合, 階差法を 用いなくてもハンケル変换を用いて政密解を求如るこ とができ，温度分布洁次式となる。

$$
\begin{aligned}
T & =-\frac{2}{\pi} \frac{q_{0}}{\lambda} \int_{0}^{\infty} p J_{0}(p r) \vec{f}(p) \int_{0}^{\infty} x g(t-s) \\
& \times \int_{0}^{\infty} \cos \xi z e^{-\kappa s\left(p^{2}+\dot{*}^{2}\right)} d \xi d s d p \\
& =-\frac{q_{0}}{\lambda} \int_{0}^{\infty} p J_{0}(p r) \vec{f}(p) \int_{0}^{\infty} x g(t-s) \\
& \times \frac{1}{\sqrt{\pi x s}} e^{-\left(\kappa s p^{2}+z^{2} / 4 \kappa s\right)} d s d p
\end{aligned}
$$

ただし, $\bar{f}(p)$ は $f(r) H(r-a) H(b-r)$ をハンケル変 鸷したものであり次式でえられる。

$$
\vec{f}(p)=\int_{a}^{b} f(r) r J_{0}(p r) d r
$$

2.2 応力解析 三次元軸対称熱空力問題法熱弹 性変位ボテンシャルゅとラプの関数Lを用いて解析 できる。のとき，然位 $u_{j}$ と夜力成分 $\sigma_{i j}$ 住

$$
\left.\begin{array}{l}
u_{r}=\Phi_{, r}-L_{, r z} \\
u_{z}=\emptyset_{. z}+2(1-\nu) \nabla^{2} L-L, z z \\
\sigma_{r r}=2 \mu\left[\Phi_{, r r}-\nabla^{2} \emptyset+\left(\nu \nabla^{2} L-L_{, r r}\right)_{, z}\right] \\
\sigma_{\theta \theta}=2 \mu\left[r^{-1} \Phi_{, r}-\nabla^{2} \Phi+\left(\nu \nabla^{2} L-r^{-1} L_{, r}\right)_{, z}\right] \\
\sigma_{z z}=2 \mu\left[\Phi_{, z z}-\nabla^{2} \Phi+\left\{(2-\nu) \nabla^{2} L-L_{, z z}\right\}_{, z}\right] \\
\sigma_{r \theta}=2 \mu\left[\Phi_{, r z}+\left\{(1-\nu) \nabla^{2} L-L_{, z z}\right\}_{, r}\right]
\end{array}\right\}
$$

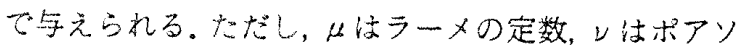

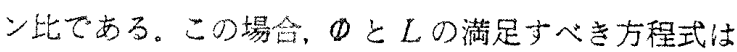

$$
\nabla^{2} \Phi=\frac{1+\nu}{1-\nu} \alpha T, \nabla^{4} L=0
$$

である。ただし， $\alpha$ は線膨倨係数である。

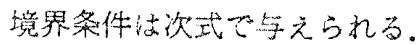

$$
\begin{aligned}
& z=0 \text { て } \quad \sigma_{r z}=0 \\
& z=0 \tau \begin{cases}u \iota_{z}=0 & (0 \leq r<a, b<r<\infty) \\
\sigma_{z z}=0 & (a<r<b)\end{cases}
\end{aligned}
$$

吾裂琵面心熱吸収一 $-q_{0} f(r) g(t)$ が存在し，かつ熱放 散がある場合、式(22)の関数形として

$$
\begin{aligned}
& \Phi=-\frac{1+\nu}{1-\nu} \alpha \frac{q_{0}}{i} \sum_{i=1}^{j} C_{j k} \sum_{n=1}^{\infty} A_{k n} x \Delta t_{k} \int_{0}^{\infty} \frac{1}{s_{k}} Z_{n}(p) J_{0}(p r) e^{-s_{k} z} d p \\
& L=\alpha \int_{0}^{\infty} \frac{1}{p^{2}}(A+p B z) J_{0}(p r) e^{-p z} d p \ldots \ldots \ldots \ldots \ldots \ldots \ldots \ldots \ldots \ldots \ldots \ldots \ldots \ldots \ldots \ldots \ldots \ldots \ldots \ldots
\end{aligned}
$$

を採用する。ただし，A，B法境照条件で決定さ机る末知数である。式(25)，(26)を式(21)心代入すると

$$
\begin{aligned}
& u_{r}=\frac{1+\nu}{1-\nu} \alpha q_{0} \frac{q_{0}}{\lambda} \sum_{k=1}^{\infty} C_{j k} \sum_{n=1}^{\infty} A_{k n} x \Delta t_{k} \int_{0}^{\infty} \frac{p}{s_{k}} Z_{n}(p) J_{1}(p r) e^{-s_{k} z} d p-\alpha \int_{0}^{\infty}[A+(p z-1) B] J_{1}(p r) e^{-p z} d p \\
& u_{z}=\frac{1+\nu}{1-\nu} \alpha \frac{q_{0}}{\lambda} \sum_{k=1}^{j} C_{j k} \sum_{n=1}^{\infty} A_{k n} x \Delta t_{k} \int_{0}^{\infty} Z_{n}(p) J_{0}(p r) e^{-s_{n} z} d p \\
& -a \int_{0}^{\infty}[A+\{2(1-2 \nu)+p z\} B] J_{0}(p r) e^{-p z} d p \\
& \sigma_{r r}=-2 \frac{1+\nu}{1-\nu} \alpha \mu \frac{q_{0}}{\lambda} \sum_{k=1}^{j} C_{j k} \sum_{n=1}^{\infty} A_{k n} x \Delta t_{k} \int_{0}^{\infty} \frac{1}{s_{k}} Z_{n}(p)\left[p r^{-1} J_{1}(p r)-s_{k}^{2} J_{0}(p r)\right] e^{-s_{k z}} d p \\
& +2 \alpha \mu \int_{0}^{\infty} p[-A+\{(1-2 \nu)-p z\} B] J_{0}(p r) e^{-p z} d p+2 \alpha \mu \int_{0}^{\infty}[A-(1-p z) B] r^{-1} J_{1}(p r) e^{-p z} d p \\
& \sigma_{\theta \theta}=2 \frac{1+\nu}{1-\nu} a \mu \frac{q_{0}}{\lambda} \sum_{k=1}^{j} C_{j k} \sum_{n=1}^{\infty} A_{k n} \int_{0}^{\infty} \frac{1}{s_{k}} Z_{n}(p)\left[x \Delta t_{k} p r^{-1} J_{1}(p r)+J_{0}(p r)\right] e^{-s_{k n} z} d p \\
& +4 \nu \alpha \mu \int_{0}^{\infty} p B J_{0}(p r) e^{-p z} d p-2 \alpha \mu \int_{0}^{\infty}(A+p B z) r^{-1} J_{1}(p r) e^{-p z} d p \\
& \sigma_{z z}=-2 \frac{1+\nu}{1-\nu} \alpha \mu \frac{q_{0}}{\lambda} \sum_{k=1}^{j} C_{j k} \sum_{n=1}^{\infty} A_{k n} x \Delta t_{k} \int_{0}^{\infty} \frac{p^{2}}{S_{k}} Z_{n}(p) J_{0}(p r) e^{-s i z z} d p \\
& +2 \alpha \mu \int_{0}^{\infty} p[A+\{(1-2 \nu)+p z\} B] J_{0}(p r) e^{-p z} d p \\
& \sigma_{r z}=-2 \frac{1+\nu}{1-\nu} a \mu \frac{q_{0}}{\lambda} \sum_{k=1}^{j} C_{j k} \sum_{n=1}^{\infty} A_{k n} x \Delta t_{n} \int_{0}^{\infty} p Z_{n}(p) J_{l}(p r) e^{-i n k z} d p \\
& +2 a \mu \int_{11}^{\infty} p[A+(-2 \nu+p z) B] J_{1}(p r) e^{-p z} d p
\end{aligned}
$$


となる。境界条件式 (23)より，

$A=2 \nu B+\frac{1+\nu}{1-\nu} \frac{q_{0}}{\lambda} \sum_{k=1}^{j} C_{j k} \sum_{n=1}^{\infty} A_{n n} \chi \Delta t_{k} Z_{n}(p)$

て衰される。式(27)を式(24)に代入し，式(28)の関係用いると，知数 $B$ 決定する方程式として次式で示され 万三重連立積分方程式を得る。

$$
\left.\begin{array}{ll}
\int_{0}^{\infty} B J_{0}(p r) d p=0 & (0 \leq r<a, b<r<\infty) \\
\int_{0}^{\infty} p B J_{0}(p r) d p=-\frac{1+\nu}{1-\nu} \frac{q_{0}}{\lambda} \sum_{k=1}^{j} C_{j k} \sum_{n=1}^{\infty} A_{k n} x \Delta t_{k} \int_{0}^{\infty} p\left(1-\frac{p}{s_{k}}\right) Z_{n}(p) J_{0}(p r) d p & (a<r<b)
\end{array}\right\}
$$

式(29)流式(9)と同じ形の積分方程式であるのて同䓠に求めると,

$$
B=-\frac{1+\nu}{1-\nu}-\frac{q_{0}}{\lambda} \sum_{m=1}^{\infty} B_{m} Z_{m}(p)
$$

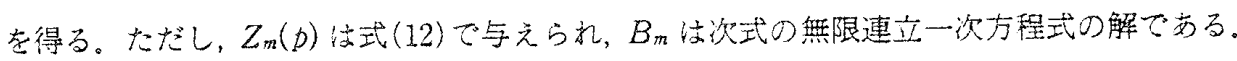

$$
\sum_{m=1}^{\infty} B_{m} \int_{0}^{\infty} Z_{m}(p) Z_{i}(p) d p=\sum_{k=1}^{j} C_{j k} \sum_{n=1}^{\infty} A_{k n} x \Delta t_{k} \int_{0}^{\infty}\left(1-\frac{p}{s_{k}}\right) Z_{n}(p) Z_{i}(p) d p \quad(i=1,2,3, \cdots)
$$

したがって，式(28)，(30)を式(27)に代入すると，z=0上の垂直応力 $\sigma_{z z}$ と開口変位 $u_{z}$ 法次式となる。

$$
\left(\sigma_{z z}\right)_{z=0}= \begin{cases}0 & (a<r<b) \\ 2 \frac{1+\nu}{1-\nu} \alpha \mu \frac{q_{0}}{\lambda}\left[\sum_{k=1}^{j} C_{j k} \sum_{n=1}^{\infty} A_{k n} x \Delta t_{k} \int_{0}^{\infty} p\left(1-\frac{p}{s_{k}}\right) Z_{n}(p) J_{0}(p r) d p\right. & \\ \left.-\sum_{m=1}^{\infty} B_{m} \int_{0}^{\infty} p Z_{m}(p) J_{0}(p r) d p\right] & (0 \leq r<a, b<r<\infty)\end{cases}
$$

$$
\left(u_{z}\right)_{z=0}= \begin{cases}0 & (0 \leq r<a, b<r<\infty) \\ \frac{4}{\pi c d}(1+\nu) \alpha \frac{q_{0}}{\lambda} \sum_{m=1}^{\infty} B_{m} \sin m \emptyset & (a<r<b)\end{cases}
$$

上って，き裂の内側先端 $(r=a)$ およで外側先端 $(r=b)$ におけるモード】の応力拡大係数 $K_{1 i}, K_{10}$ はそれぞれ

$$
K_{1 i}=\frac{\mu}{1-\nu} \lim _{r \rightarrow a+} \sqrt{\frac{\pi}{2(r-a)}}\left(u_{z}\right)_{z=0}, K_{10}=\frac{\mu}{1-\nu} \lim _{r \rightarrow b-} \sqrt{\frac{\pi}{2(b-r)}}\left(u_{z}\right)_{z=0}
$$

て定義され，

$$
K_{1 i}=\frac{4 \sqrt{a}}{c d \sqrt{\pi c d}} \frac{1+\nu}{1-\nu} \alpha \mu \frac{q_{1}}{\lambda} \sum_{m=1}^{\infty} B_{m} m, K_{10}=\frac{4 \sqrt{b}}{c d \sqrt{\pi c d}} \frac{1+\nu}{1-\nu} \alpha \mu \frac{q_{0}}{i} \sum_{m=1}^{\infty}(-1)^{m+1} B_{m} m
$$

を得る。

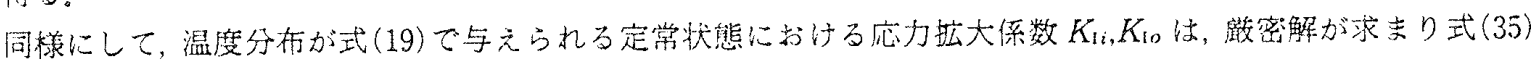

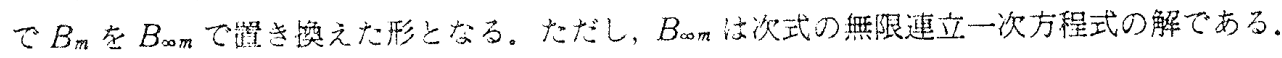

$$
\sum_{m=1}^{\infty} B_{\infty m} \int_{0}^{\infty} Z_{m}(p) Z_{i}(p) d p=\sum_{n=1}^{\infty} A_{\infty n} \int_{0}^{\infty} \frac{1}{p^{2}} Z_{n}(p) Z_{i}(p) d p \quad(i=1,2,3, \cdots)
$$

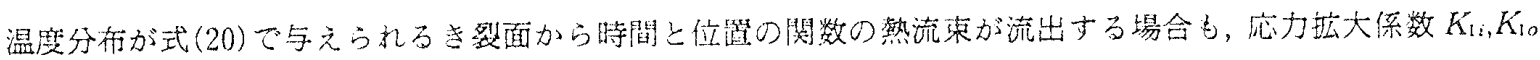

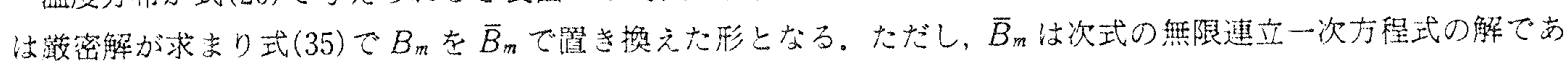
る。

$$
\sum_{m=1}^{\infty} \bar{B}_{m} \int_{0}^{\infty} Z_{m}(p) Z_{i}(p) d p=\int_{0}^{\infty} p \bar{f}(p) Z_{i}(p) \int_{0}^{t} x g(t-s) \operatorname{erfc}(p \sqrt{x s}) d s d p \quad(i=1,2,3, \cdots)
$$

たたし， erfc（）は次式て表きれる余垶美関数である。

$$
\operatorname{erfc}(x)=\frac{2}{\sqrt{\pi}} \int_{x}^{\infty} e^{-y^{2}} d y
$$

\section{3. 数值計算綰果と考察}

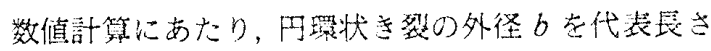
にとり次式で示される照次元主老等入する。

$$
\begin{aligned}
& \rho=r / b, \quad \zeta=z / b, \quad \gamma=a / b, \\
& t^{\prime}=x t / b^{2}, \quad B_{i}=b h, \quad \hat{T}=T / \theta_{0},
\end{aligned}
$$

$$
\overrightarrow{K_{3}}=K_{I} /\left\{2(1+\nu) \alpha \mu \sqrt{b} \theta_{0} /(1-\nu)\right\}
$$

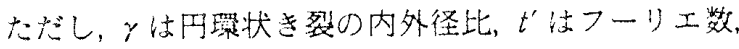
$B_{i}$ はビオ数, $\theta_{0}=b q_{0} / \lambda$ である。

この解法の妥当性と式(35)の $m$ に関与る収染性の 吟味のため，晸密解が得られる $B_{i}=0$ の場合について 


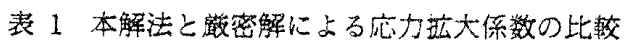
および $m$ に関与る収束性

\begin{tabular}{|c|c|c|c|c|c|}
\hline$M$ & 0.1 & 1.0 & 10.0 & 100.0 & $\infty$ \\
\hline 5 & 0.059 & 0.142 & 0.208 & 0.232 & 0.245 \\
\hline 10 & 0.059 & 0.142 & 0.209 & 0.233 & 0.245 \\
\hline R.S. & 0.059 & 0.142 & 0.209 & 0.234 & 0.245 \\
\hline
\end{tabular}

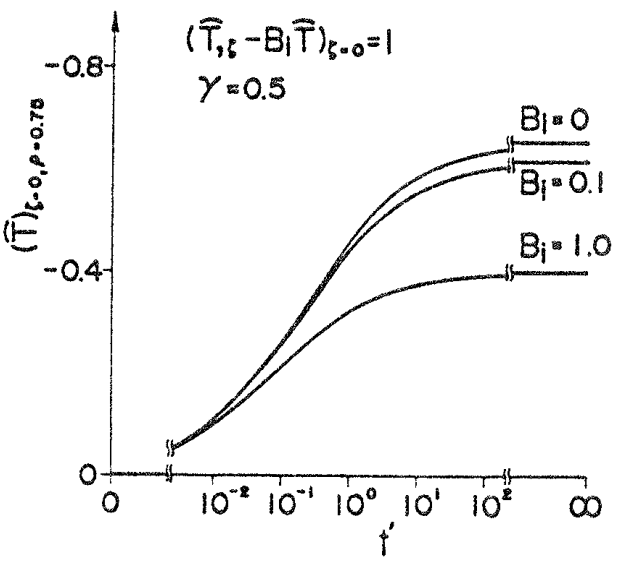

(吸收熱㨁がー $q_{0} f(r) g(t)=-q_{0}$ で与えられる場合)

図 2 温度 $(\bar{T})_{\xi=0, \rho=0.75}$ の時間溶化

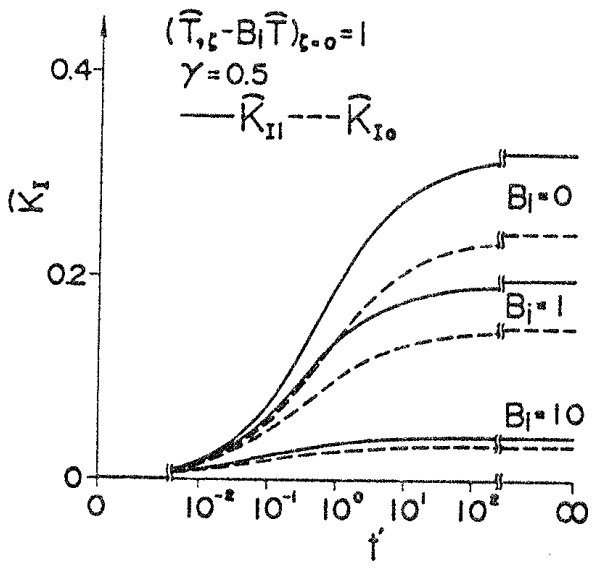

(吸収熟站がー $q_{0} f(r) g(t)=-q_{0}$ て与えっれる場合)

図 3 無次元応力㧨大係数 $\bar{K}, \infty$ 時間受化

外側き裂先端の応力拡大係数 $K_{10}$ 計算し，その結果 を表 1 に示す.ここて， $\gamma=0.5$, 熱吸収 $-q_{0} f(r) g(t)=$ 一和とした。表1において $M$ 洁式(35)中の $\sum_{m=0}^{\infty} \sum_{m=0}^{M}$ て打ち切ったときの上限の值である。解の収束性につ いては：M=10（ $\gamma=0.5$ の場合）を採用すれば十分であ ることがわかる。本解法の棨当性心ついては，各フ一 リ工数に効し本解法に上る応力抬大係数が旋密解と一 致しているこ上により，本解法により十分精度のよい

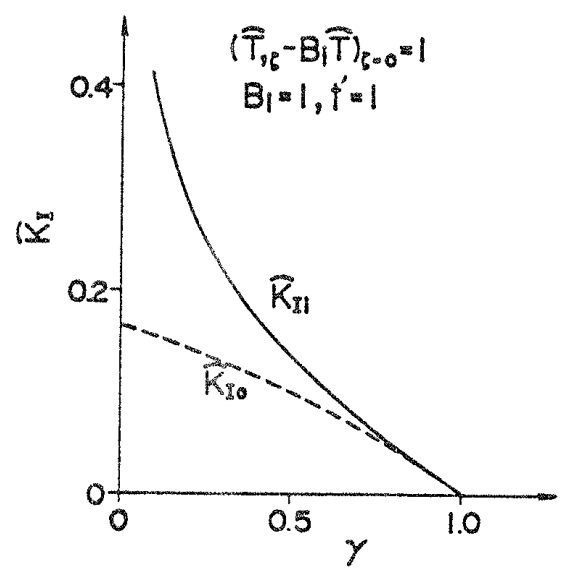

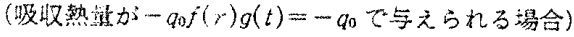

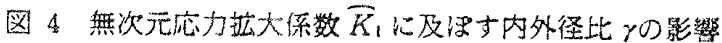

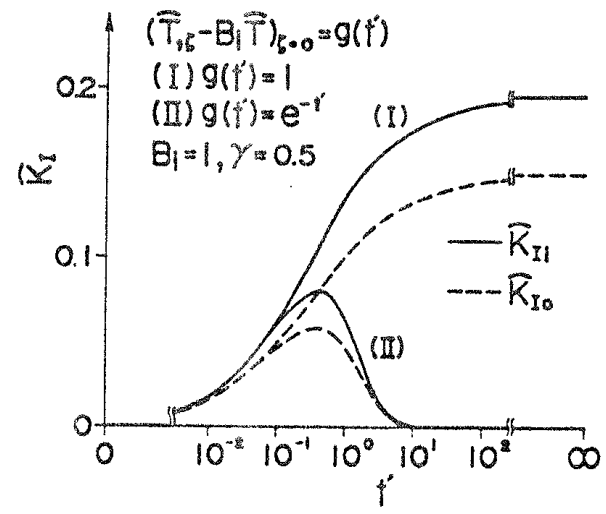

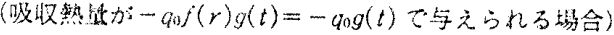

図 5 無次元沁力抬大係数 $\bar{K}_{1}$ の時間変化

解が得られることがかかる。

図 2 に吸収熟黒がー $q_{0} f(r) g(t)=-q_{0}$ で与えられる 場合の温度 $(\widehat{T})_{\zeta=0, \rho=1, ; 5}$ の時間変化を示寸.フーリ土 数が大きくなる活ど，また，ビオ数が小さくなるほど 温度は低下していることがわかる。

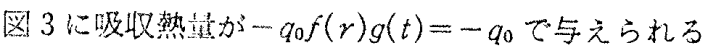
場合の無次元応力蛙大係数 $\widehat{K}_{1}$ の時間変化を示す，無

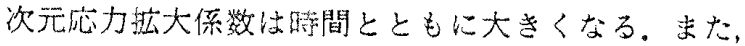
ビオ数が小さくなるによ゙大きくなっっていることがわか

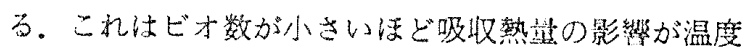
変化仙上り大きく影到するた好上考えられる。また， $\widehat{K}_{1 i}$ 好に $\widehat{K}_{10}$ より大きいことがわかる。

图 4に吸収熱望がー $q_{0} f(r) g(t)=-q_{0}$ で与えられる 場合の無次元应力抬大係数 $\widehat{K}_{1}$ と内外径比 $\gamma$ との関倸 を示す。琶裂の内半径が大きくなるとともに辰 $\widehat{K}_{10}$ の值证近づき,内半径が小さくなるとともに $\widehat{K}_{1 i}$ の値

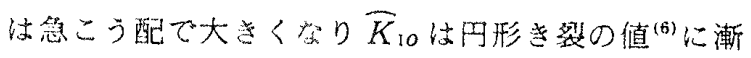
近する。茫た， $\widehat{K}_{1 i}$ は极に $\widehat{K}_{10}$ 上り大きいことがわ 
かる。

図 5 亿吸収熱量がー- $q_{0} f(r) g(t)=-q_{0} g(t)$ で与えら 机る場合の無次元応力㹡大係数 $\widehat{K}_{1}$ の時間変化を示

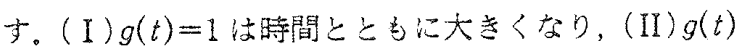
$=e^{-t^{\prime}}$ 结(I)とともに增加し最大值を示したのち減少 して零となることがわかる。志た， $\widehat{K}_{1 i}$ 流つ权化 $\widehat{K}_{10}$ より大きいことがかかる。

\section{4. 結論}

き裂害面に時間と位置により変化する熱吸収が存在 する円環状き裂を有する無限体の非定常熱応力を解析 し，与えられた熱的就よで力学的境界条体のもとに数 值計算し, 次の結諭得志。

（1）著者らによって示された時間代対して踏差法 を用い空間变数心対し解析解得万方法か，当裂表面 亿時間上位監に上り变化する熱吸收が存在する円環状 き裂を有する無限体の非定常熱応力の解析に有効な解 析法であることを示しな。
（2）無次元沁力搪大係数はビオ数が小さい添ど大 きくなる。また，熟吸収を位置のみの関数とした場合， 応力搪大係数洨フーリ工数が大きくなる活ど大きな゙值 を示す。

（3）熱吸収が時間のみの関数で与えうれる場合, き製内側の応力抬大保数估つ称にき裂外側の応力拉大 係数上り大きくなる。終わりに，本研究の遂行にあた

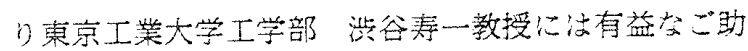
言をいたな゙きこここ記して訲礼申し上代ます。

\section{文塥}

(1) 小沓・丹羽, 策論, 43-366(昭 52), 442.

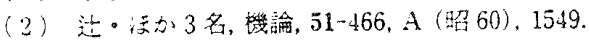

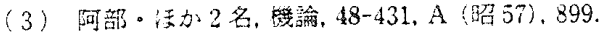

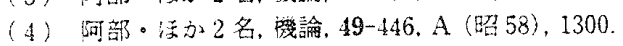

（5）野田・科保，幾論，51-162, A (眧 60)，463.

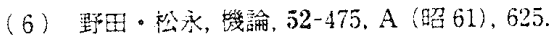

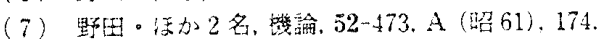

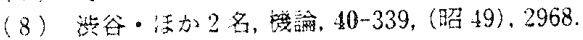

